7) Toutiousity; Resection with failed anastomosis. 8) Stenosis caused by atherosclerosis; Resection of atheromatous plaque, stripping of vertebral and subclavian artery. 9) "Spasm" (Irregularity of vertebral wall showed by angiography in turning of head); Stripping of vertebral artery.

\title{
84. Surgical Treatment for Carotid Spasm in the Neck
}

\author{
Jiro Suzuki, Akira TAKaku, \\ Division of Neurosurgery, Institute of Brain Diseases, \\ Tohoku University School of Medicine \\ Ryunchan KwaK, Teruhiko TANAKA and Itaru OHARA \\ 2nd Surgical Clinic, Tohoku University School of Medicine
}

There have been some reports indicating that angiospasm may occur in the cerebral vessels of man and causes brain disorder. However, none of them has observed the spasm under direct observation.

Recently, we identified surgically four patients as the angiospasm of internal carotid artery in the neck. The patients had various complaints suggesting brain disorders. In all cases, there were closed cervial craninal trauma in their past histories. Angiographic examination revealed nonfilling or narrowing of the internal carotid artery which commonly occur at sites about two $\mathrm{cm}$ rostral to the carotid bifurcation.

After surgical treatment using our carotid dilatation method, the complaint were improved in parallel with angiographic disappearance of the narrowing. This may suggest that the angiospasm occures in the internal carotid artery of the neck. The internal carotid spasm in the neck would cause some types of more long lasting organic carotid occlusion.

\section{Etiologic Consideration of the Cases with Malformed Vascular Anomalies at the Base of the Brain}

\author{
Shinichiro Endo, Ichiro OGAwA, Yaichi AKIBA, \\ Kenshichiro ENDo and Toshio YAMADA \\ 2nd Dept. of Surgery, Fukushima Medical College
}

4 cases reported here belong to a series of cases with markedly

$$
-218-
$$

